\title{
Magnetic Resonance Imaging Urodynamics. Technique Development and Preliminary Results
}

\author{
Gustavo Borghesi, Rogerio Simonetti, Suzan M. Goldman, Jacob Szejnfeld, Miguel Srougi, \\ Valdemar Ortiz, Homero Bruschini
}

Department of Urology, Federal University of Sao Paulo, UNIFESP, Sao Paulo, SP, Brazil

\begin{abstract}
Objectives: In this preliminary study we report the development of the video urodynamic technique using magnetic resonance imaging (MRI).

Materials and Methods: We studied 6 women with genuine stress urinary incontinence, diagnosed by history and physical examination. Urodynamic examination was performed on multichannel equipment with the patient in the supine position. Coughing and Valsalva maneuvers were performed at volumes of 150, 250 and $350 \mathrm{~mL}$. Simultaneously, MRI was carried out by using 1.5 T GE Signa CV/i high-speed scanner with real time fluoroscopic imaging possibilities. Fluoroscopic imaging was accomplished in the corresponding planes with T2-weighted single shot fast spin echo sequences at a speed of about 1 frame per second. Both studies were recorded and synchronized, resulting in a single video urodynamic examination. Results: Dynamic MRI with cine-loop reconstruction of 1 image per second demonstrated the movement of all compartment of the relaxed pelvis during straining with the concomitant registration of abdominal and intravesical pressures. In 5 patients, urinary leakage was demonstrated during straining and the Valsalva leak point pressure (VLPP) was determined as the

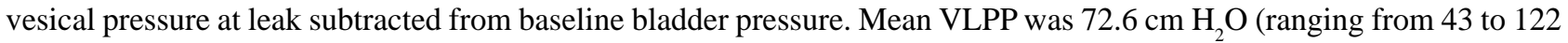
$\mathrm{cm} \mathrm{H}_{2} \mathrm{O}$ ).

Conclusions: The concept of MRI video urodynamics is feasible. In a clinical perspective, practical aspects represent a barrier to daily use and it should be recommended for research purposes.
\end{abstract}

Key words: urinary incontinence, stress; urodynamics; magnetic resonance imaging

Int Braz J Urol. 2006; 32: 336-41

\section{INTRODUCTION}

Video urodynamics displaying simultaneous data with radiographic images of the bladder and urethra were originally described by Miller in 1971 (1). Pressure flow study combined with fluoroscopy or ultrasonography is generally referred as video urodynamics, which is currently the gold standard to diagnose and localize lower urinary tract dysfunction (2).
Magnetic resonance imaging (MRI) with its noninvasive, nonionizing, multi-planner imaging capabilities offers distinct advantages over computerized tomography/fluoroscopy and since it has greater accuracy over ultrasound in the detection of discrete structures, it has rapidly become a major diagnostic tool in the assessment of pelvic conditions.

Our attention was prompted by the possibility of using this new modality towards a better understanding of female stress urinary incontinence 
(SUI). SUI is the observation of involuntary leakage of urine from the urethra synchronous with exertion/ effort, or sneezing, or coughing (3). Normally, anatomical support of the bladder neck and proximal urethra allows the transmission of increased intraabdominal pressure to that area of continence, compensating the closure mechanism and maintaining continence. Leakage occurs as a result of pressure transmission failure, lack of intact intrinsic mechanism or both (4). However, these pathophysiological concepts are yet a matter of controversy.

To this date, there has been no satisfactory investigative method combining observations regarding bladder and urethra hypermobility, intrinsic sphincter insufficiency, and anatomical pelvic floor abnormalities in such patients. The fast MRI-systems available have a capability of functional examinations. Those have been explored and are currently used for evaluating other organ systems. The possibility of simultaneous evaluation of bladder and abdominal pressures, bladder and urethral mobility under effort, and pelvic floor muscles integrity and disposition, may have a potential role for better understanding SUI (5). Herein we report this preliminary and innovative effort to develop this technique.

\section{MATERIALS AND METHODS}

After our Institutional Review Board's approval and informed consent, we studied 6 women with genuine stress urinary incontinence, diagnosed by history and physical examination. Mean age was 53.1 years (range 28 to 70 ).

Urodynamic examination was performed on multichannel equipment with the patient in the supine position. Intravesical pressure was measured by a transurethral 7F double lumen catheter. Abdominal pressure was measured by a balloon catheter inflated with 5 to $10 \mathrm{~mL}$ saline. Detrusor pressure was indicated by electronically subtracting abdominal pressure from bladder pressure. Filling cystometry was performed at filling rate of $50 \mathrm{~mL}$ per minute with saline at room temperature. Coughing and Valsalva maneuvers were performed at volumes of 150,250 and $350 \mathrm{~mL}$.
Simultaneously, magnetic resonance imaging was performed by using $1.5 \mathrm{~T}$ (Magneton Sonata Maestro Class, Siemens, Erlangen, Germany) with a 6-channel belt type surface coil placed around the patient at the level of the symphysis pubis. Morphological T2 true FISP (true fast imaging with steady-state precession TR 4.5, TE 2.3) weighted images were taken in sagittal plane during 360 seconds at a speed of 1 frame per second.

Some devices were developed to accomplish the MRI urodynamics. An especially designed portable polygraph was coupled to a notebook, together with an adjustable infusion pump. Tube connections 5 meters long were adapted to the system, in order to maintain the electronic equipments out of the reach of the magnetic field.

Both studies were recorded and synchronized, resulting in a single video urodynamic examination (Figure-1).

\section{RESULTS}

This is the report of the last 6 patients when synchronization of both exams was achieved. Previously, 8 patients were submitted to the MRI video urodynamics with unsatisfactory results. Although both MRI and urodynamic data were properly obtained, they were not synchronous making impossible their evaluation as a single video urodynamic exam.

Mean age was 53.1 years (range 28 to 70 ). Table-1 shows patients characteristics.

Dynamic MRI with cine-loop reconstruction of 1 image per second produced images of striking vividness, which demonstrated the movement of all compartment of the relaxed pelvis during straining with the concomitant registration of abdominal and intravesical pressures (Figure-2). In 5 patients, urinary leakage was demonstrated during straining and the Valsalva leak point pressure (VLPP) was determined as the vesical pressure at leak subtracted from baseline bladder pressure. Mean VLPP was $72.6 \mathrm{~cm} \mathrm{H} \mathrm{H}_{2} \mathrm{O}$ (ranging from 43 to $122 \mathrm{~cm} \mathrm{H}_{2} \mathrm{O}$ ) (Figure-2). The recording of leaking point pressure values simultaneous to the anatomic images of pelvic floor 


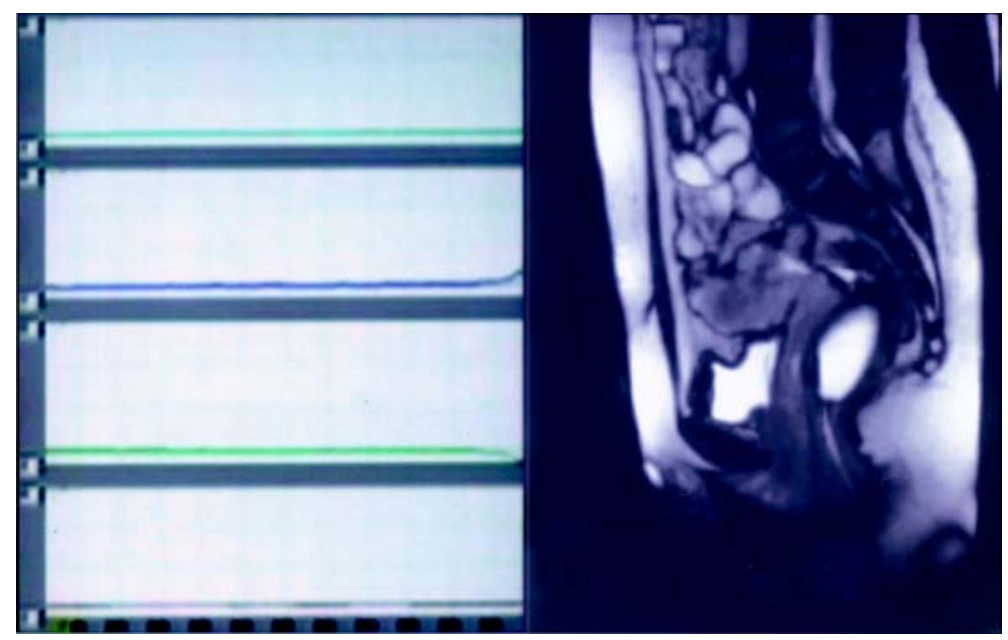

Figure 1 - Magnetic resonance imaging video urodynamics. Synchronous display of urodynamic parameters and sagittal magnetic resonance image of the pelvis.

Table 1 - Patient's history and physical examination characteristics.

\begin{tabular}{cccccc} 
Patient 1 & Patient 2 & Patient 3 & Patient 4 & Patient 5 & Patient 6 \\
\hline 50 & 51 & 28 & 70 & 70 & 50 \\
none & 4 & 3 & 3 & 5 & 4 \\
none & none & 2 & 3 & 4 & none \\
none & none & none & none & none & none \\
I & I & I & absent & I & absent \\
absent & absent & absent & absent & absent & absent \\
present & present & present & absent & present & present
\end{tabular}

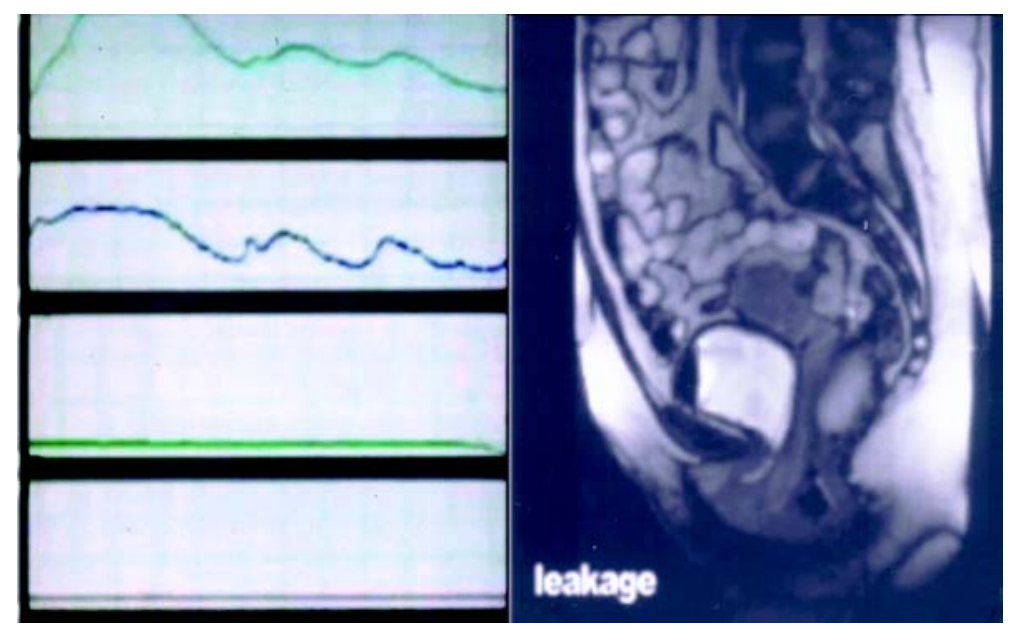

Figure 2 - Magnetic resonance imaging video urodynamics. Urodynamic parameters during Valsalva maneuvers concomitant to magnetic resonance image of the pelvis showing descent of the bladder neck below the pucococcygeal line and the urethra fully opened, allowing loss of urine. 
muscles and bladder base descent under effort was achieved, providing a new visual dimension to understand SUI.

Urinary leakage was not demonstrated during MRI urodynamics in only one patient, despite previously demonstrated by physical examination. No pelvic organ prolapse was observed in this patient, neither by physical examination nor by MRI. We cannot doubtless state the reason for this disagreement, but possibly the patient's inability to satisfactorily increase intra-abdominal pressure during cough and Valsalva maneuvers. Patient age (70 years old), and the performance of the exam in the supine position inside the MRI tunnel with a belt type coil placed around the patient's body may have played a role as well.

The pubococcygeal reference line was employed for diagnosis of descent of the organs. Cystocele was seen in all patients (4 mild and 2 moderate) and rectocele in only one (moderate). No enterocele was noted. Pelvic organ prolapse evaluation by MRI correlated well with the physician examination, except in one patient. Table- 2 shows the pelvic organ prolapse findings by the 2 methods.

Patients accepted well the procedure and experienced discomfort similar to standard urodynamic test. All patients said that they would agree to have a similar procedure in the future.

\section{COMMENTS}

Recent studies have changed our understanding of underlying multifactorial causes to incontinence. Our first understandings supposed that SUI was mainly a result of descent of the proximal urethra in relation to pelvic floor. Lately, our attention was focused to the fact that in many women with bladder dysfunction disorders including incontinence, other facts have to be considered as specific damage to pelvic muscle ligaments, integrity of soft connective tissue, peripheral nerves and segmental vessels.

Pelvic floor weakness strongly correlates with lower urinary tract dysfunction. Stress urinary incontinence often coexists with pelvic organ prolapse and vice versa. The fact that pelvic muscle exercises improve urinary control in many women confirms that muscle action can influence urinary control. The lack of universal success of such therapy emphasizes the importance of a better understanding of the relationship among pelvic muscles, pelvic floor fascial structures, bladder and urethra mobility, and how their damage can lead or contribute to SUI. Most women with SUI seem to have a combination of urethral dysfunction and loss of support, and scientific study of these issues awaits insight into the quantification of each of these parameters as independent variables $(6,7)$.

In the last 10 years there has been a great increase in both availability and quality of MRI examinations. Its potential in the evaluation of pelvic floor disorders is well established. The advantages of MRI are well known and include the lack of radiation, and the ability to provide a high-resolution global assessment of the pelvis, its constituent organs, and the musculofascial support structures. Analysis of the levator plate complex can be made in the axial and mid-coronal images. Increased signal intensity

Table 2 - Pelvic organ prolapse findings.

\begin{tabular}{lcccc}
\hline & Physical Examination & (grades I to IV) & \multicolumn{2}{c}{ MRI (mild, moderate, severe) } \\
\hline & cistocele & rectocele & cistocele & rectocele \\
Patient 1 & I & absent & mild & absent \\
Patient 2 & I & absent & mild & absent \\
Patient 3 & I & absent & mild & absent \\
Patient 4 & absent & absent & mild & absent \\
Patient 5 & I & absent & mild & absent \\
Patient 6 & absent & absent & moderate & moderate \\
\hline
\end{tabular}


of the levator relative to the obturator internus muscle on proton density images, decreased length, thickness and muscle area can be used as parameters of levator complex lesion $(8,9)$. Fascial defects cannot be appreciated on functional cine MRI alone (10). Endoluminal imaging has the potential to solve the problem regarding urethral support because a smaller field of view can be used, thereby providing images of higher spatial resolution. However, it would interfere with the synchronous realization of the urodynamics.

Functional MRI of the pelvic floor with depiction of organ movement was first introduced by Yang et al. and Kruyt et al. in 1991. (11,12). Nevertheless, MRI functional examinations are still limited in urological practice. The combination of function and morphology allows for an innovative view of the pelvic floor, and thus adds to our understanding of the various structure interactions. The intention of developing a MRI urodynamic examination is the possibility of gathering the objective functional test of bladder and urethra function provided by urodynamics with the best anatomical images of the pelvis offered by MRI. However, this was initially hampered by some practical problems. The magnetic fields prevent the use of other magnetic material close to the patient. We had to develop long connections to keep the polygraph outside the room. The long tunnel in which patients were placed was an obstacle to routine functional examinations, like having to perform micturition lying on the table within the MRI. Dynamic MRI has been performed previously during voiding only in an open MRI system (7). The problems with open systems are costs and availability, as well as poor spatial and time resolution compared with closed systems (13). For this reason, in this study, pressure/ flow studies were not performed.

Despite the fact that MRI is considered to be physically benign, it appears to be associated with psychological side effects. Patients may experience severe claustrophobia or panic attacks and others may report milder distress due to the necessity to lie in a very confined space for a long period. Although all patients in this study have undergone a MRI procedure for the first time, none of them experienced significant anxiety. Still, they have not been evaluated with pre and post-scan specific anxiety questionnaires.

To our knowledge, these preliminary results represent the first attempt where dynamic MRI has been used during urodynamic examination.

\section{CONCLUSIONS}

The concept of MRI video urodynamics is feasible and the basic technique was achieved after studying the 6 first patients. It is likely to believe that difficulties in some practical aspects represent a barrier to daily use, thus limiting routine clinical studies. However, it brings new possibilities for the study of SUI and bladder dysfunction and represents a useful tool for research purposes and further studies. Clinical research is needed to evaluate the possible diagnostic gains and possible additional use of MRI in urology.

\section{CONFLICT OF INTEREST}

None declared.

\section{REFERENCES}

1. Miller ER: Combined Monitoring for the Study of Continence and Voiding. In: Hinman F (ed.), Hydrodynamics of Micturition. Springfield, Charles C. Thomas. 1971; pp. 5-17.

2. Chancellor M, Blaivas J: Synchronous PressureUroflow and Video-Urodynamics. In: Blaivas J, Chancellor M (ed.), Atlas of Urodynamics. Philadelphia, Williams \& Wilkins, 1996; pp. 88-104.

3. Abrams P, Cardozo L, Fall M, Griffiths D, Rosier P, Ulmsten $\mathrm{U}$, et al.: The standardisation of terminology in lower urinary tract function: report from the standardisation sub-committee of the International Continence Society. Urology. 2003; 61: 37-49.

4. Klutke C, Golomb J, Barbaric Z, Raz S: The anatomy of stress incontinence: magnetic resonance imaging of the female bladder neck and urethra. J Urol. 1990; 143: 563-6.

5. Mostwin JL, Genadry R, Saunders R, Yang A: Stress incontinence observed with real time sonography and 
dynamic fastscan magnetic resonance imaginginsights into pathophysiology. Scand J Urol Nephrol Suppl. 2001: 94-9; discussion 106-25.

6. DeLancey JO: Stress urinary incontinence: where are we now, where should we go? Am J Obstet Gynecol. 1996; 175: 311-9.

7. Hedlund H, Bo K, Lilleas F, Talseth T, Tillung T: The clinical value of dynamic magnetic resonance imaging in normal and incontinent women — a preliminary study on micturition. Scand J Urol Nephrol Suppl. 2001: 8791; discussion 106-25.

8. Tunn R, Paris S, Fischer W, Hamm B, Kuchinke J: Static magnetic resonance imaging of the pelvic floor muscle morphology in women with stress urinary incontinence and pelvic prolapse. Neurourol Urodyn. 1998; 17: 579-89.

\author{
Correspondence address: \\ Dr. Homero Bruschini \\ Rua Barata Ribeiro 414 / 35 \\ São Paulo, SP, 01308-000, Brazil \\ Fax: + $55113218-8282$ \\ E-mail: bruschini@uol.com.br
}

\section{EDITORIAL COMMENT}

The authors describe a merging of magnetic resonance imaging and urodynamic technologies. Though fluorourodynamics when initially described was potentially looked upon as an intellectual curiosity, it has since improved into a state-of-the-art method for evaluation of voiding dysfunction. Perhaps with this article we are also observing the emergence of a new marriage of technologies to aid the diagnosis and quantification of bladder dysfunction. Magnetic resonance imaging is already finding a place in the evaluation of pelvic floor prolapse. This extension of application to voiding dysfunction seems to be a reasonably logical step and in retrospect may even
9. Pannu HK: Magnetic resonance imaging of pelvic organ prolapse. Abdom Imaging. 2002; 27: 66073.

10. Lienemann A, Fischer T: Functional imaging of the pelvic floor. Eur J Radiol. 2003; 47: 117-22.

11. Yang A, Mostwin JL, Rosenshein NB, Zerhouni EA: Pelvic floor descent in women: dynamic evaluation with fast MR imaging and cinematic display. Radiology. 1991; 179: 25-33.

12. Kruyt RH, Delemarre JB, Doornbos J, Vogel HJ: Normal anorectum: dynamic MR imaging anatomy. Radiology. 1991; 179: 159-63.

13. Geitung JT: Magnetic resonance imaging of the pelvic floor-possibilities and present status. Scand J Urol Nephrol Suppl. 2001: 92-3; discussion 10625 .

Accepted after revision: April 3, 2006

be thought of as the obvious natural sequence of diagnostic evolution. It will be unusual if other research groups choose not to examine this process and thus leave these authors known for their sui generis method of patient evaluation. The authors should be credited with their original thoughts and their desire to advance science.

Dr. Steven P. Petrou

Associate Professor of Urology

Associate Dean, Mayo Clinic College of Medicine Jacksonville, Florida, USA

E-mail: petrou.steven@mayo.edu 